\title{
Pre-synaptic sympathetic calcium channels, cyclic nucleotide-coupled phosphodiesterases and cardiac excitability
}

\author{
Dan Li and David J. Paterson
}

Burdon Sanderson Cardiac Science Centre, Department of Physiology, Anatomy and Genetics, Sherrington Building, University of Oxford, Parks Road, Oxford OX1 3PT, UK

\section{Correspondence:}

\section{Dan Li \& David J. Paterson}

Department of Physiology, Anatomy and Genetics

University of Oxford

Sherrington Building,

Parks Road,

Oxford, OX1 3PT, UK

Tel: +44 1865272547 Fax: +44 1865 282453,

E-mail: dan.li@dpag.ox.ac.uk; david.paterson@dpag.ox.ac.uk 


\begin{abstract}
In sympathetic neurons innervating the heart, action potentials activate voltage-gated $\mathrm{Ca}^{2+}$ channels and evoke $\mathrm{Ca}^{2+}$ entry into presynaptic terminals triggering neurotransmitter release. Binding of transmitters to specific receptors stimulates signal transduction pathways that cause changes in cardiac function. The mechanisms contributing to presynaptic $\mathrm{Ca}^{2+}$ dynamics involve regulation of endogenous $\mathrm{Ca}^{2+}$ buffers, in particular the endoplasmic reticulum, mitochondria and cyclic nucleotide targeted pathways. The purpose of this review is to summarize and highlight recent findings about $\mathrm{Ca}^{2+}$ homeostasis in cardiac sympathetic neurons and how modulation of second messengers can drive neurotransmission and affect myocyte excitability in cardiovascular disease. Moreover, we discuss the underlying mechanism of abnormal intracellular $\mathrm{Ca}^{2+}$ homeostasis and signaling in these neurons, and speculate on the role of phosphodiesterases as a therapeutic target to restore normal autonomic transmission in disease states of overactivity.
\end{abstract}

Key words: calcium; sympathetic neuron; cyclic nucleotide; phosphodiesterase; cardiac excitability. 


\section{Introduction}

Calcium is universally regarded as a significant intracellular messenger with wide-ranging physiological roles. In the early 1880s, Sydney Ringer first realized that extracellular $\mathrm{Ca}^{2+}$ was critical for frog heart contraction[1]. Subsequent work revealed that numerous cell biological processes, including muscle contraction, transmitter release, enzyme activation, metabolism, and hormone secretion are controlled by calcium[2]. In particular, calcium is of critical importance to neurons since it participates in the transmission of the depolarizing signal and contributes to synaptic activity[3]. Various stimuli, such as changes in membrane potential, extracellular signaling molecules, or intracellular messengers, promote an increase in intracellular calcium concentration $\left(\left[\mathrm{Ca}^{2+}\right]_{\mathrm{i}}\right)$. This increase results from either the influx of extracellular $\mathrm{Ca}^{2+}$ via plasma membrane $\mathrm{Ca}^{2+}$ channels or the release of $\mathrm{Ca}^{2+}$ from internal stores, such as sarco-/endoplasmic reticulum (SR/ER) and mitochondria[4].

Two important intracellular second messengers, cyclic adenosine monophosphate (cAMP) and cyclic guanosine monophosphate (cGMP) can modulate intracellular calcium homeostasis and signaling. These cyclic nucleotides predominately exert their physiological effects via protein kinase A (PKA)- and protein kinase G (PKG)-dependent phosphorylation. cAMP and cGMP act via PKA and PKG to cause activation and inhibition of the channel current respectively[5, 6]. This occurs either via direct phosphorylation of the channels [7, 8] or through cross-play between the two cyclic nucleotides pathways via phosphodiesterases (PDEs), a group of enzymes that are involved in their hydrolysis [9-14]. cAMP and cGMP also act to terminate their own signals by activating protein phosphatases that dephosphorylate downstream targets (Figure 1).

It is well established that the autonomic nervous system is a major regulator of cardiac function, particularly during stress and exercise. Autonomic activation alters not only heart rate, conduction, and hemodynamics, but also cellular and subcellular properties of individual myocytes[15]. The action potential that arrives at the prejunctional sympathetic varicosity activates voltage-dependent calcium channels and release of neurotransmitter from vesicles. Norepinephrine (NE), the main neurotransmitter of the sympathetic nervous system, diffuses across the neuromuscular cleft and binds to $\beta$-receptors to initiates a signaling cascade to regulate cardiomyocyte excitationcontraction coupling[16]. The opposing parasympathetic branch of the autonomic nervous system is regulated by acetylcholine, which reduces contractility and heart rate via activation of muscarinic cholinergic receptors and the inhibitory $G$ protein transduction pathway[17]. The dynamics and modality of communication between neurons and cardiomyocytes determines how neuronal information is conveyed to the heart.

Autonomic dysfunction, characterized by suppressed parasympathetic activity and increased sympathetic activity, correlates with various cardiovascular pathologies that can reside at the end organ. Interestingly, this neural phenotype is coupled to impaired cyclic nucleotide pathways linked to abnormal intracellular calcium handing[18-21], and can contribute to several cardiovascular diseases such as heart failure[22, 23], arrhythmia[15], ischemia/reperfusion injury[24] and hypertension[25, 26]. Here we briefly review emerging data on how cardiac sympathetic innervation can drive myocyte excitability in cardiovascular disease. Moreover, we discuss the underlying mechanism of abnormal intracellular $\mathrm{Ca}^{2+}$ homeostasis and signaling in cardiac sympathetic neurons, and speculate on the role of PDE-coupled cyclic nucleotides as a therapeutic target to restore normal autonomic transmission. 


\section{Importance of cardiac sympathetic innervation in cardiovascular disease}

The heart is innervated by an exquisitely complex neural network. Throughout the human heart, numerous cardiac ganglia, each of which contain 200 to 1000 neurons, form synapses with the sympathetic and parasympathetic fibers that enter the pericardial space[27, 28]. The majority of sympathetic postganglionic axons innervating the heart originate from cell bodies in the stellate ganglion[29], or the caudal cervical ganglia (inferior and middle). It is well established that cardiac sympathetic hyperactivity is an early hallmark of cardiac arrhythmia[15], heart failure[30, 31], post myocardial infarction[32] and hypertension; both in humans [33, 34] and in the spontaneously hypertensive rat (SHR) [35, 36]. Whilst beta adrenergic blockade has been a mainstay therapy, its long term efficacy is still sub-optimal given off target effects[37]. Given the encouraging results of bilateral sympathectomy to treat some classes of arrhythmia[38], there has been a renewed interest to target autonomic nerves.

High cardiac sympathetic drive promotes myocyte calcium influx, increases the inotropic state of the heart, and increases myocardial oxygen demand. This can exacerbate the harmful effects of preexisting cardiac ischemia and precipitate life-threatening ventricular arrhythmias[39, 40]. Excessive adrenergic activity is unsurprisingly a negative prognostic indicator post myocardial infarction[41, 42] and during congestive cardiac failure[43, 44]. In addition, overactivity of the sympathetic nervous system in hypertension causes enhanced neurohumoral activation[45, 46] , resulting in abnormal calcium signaling in stellate neurons[25] and cardiac pacemaker cells[47]. Increased cardiac NE release[48, 49] and impaired neuronal NE reuptake[48, 50] leads increased stellate ganglion activity resulting in abnormal ECGs[51] and predisposition to arrhythmias[5254].

To fully understand the importance of sympathetic neurotransmission on end-organ function and how it might be altered in disease[55], we recently developed a model system to study the peripheral neuro-cardiac axis itself, rather than individual cells in isolation[56]. We show that sympathetic neurons are powerful drivers of myocyte cAMP activity in cardiovascular disease[56]. By cross-culturing healthy neurons onto diseased heart cells we could rescue the abnormal myocyte cAMP phenotype. Moreover, we could partially recapitulate the diseased myocyte response by culturing diseased neurons onto healthy heart cells, illustrating the impact of the neuron alone to drive cardiac excitability (Figure 2).

\section{Abnormal intracellular $\mathrm{Ca}^{2+}$ homeostasis and signaling in cardiac sympathetic neurons}

\subsection{Dysregulation of presynaptic neuronal $\mathrm{Ca}^{2+}$ handling (membrane and cytoplasm)}

In a presynaptic terminal, an action potential opens voltage-gated $\mathrm{Ca}^{2+}$ channels, and transiently increases the local $\mathrm{Ca}^{2+}$ concentration. This triggers $\mathrm{Ca}^{2+}$ release from the ER and facilitates synaptic vesicle exocytosis, thus initiating synaptic transmission. This fundamental mechanism was discovered in pioneering work on the neuromuscular junction by Katz and Miledi in 1967[57]. Cytoplasmic $\left[\mathrm{Ca}^{2+}\right]$ is maintained at low levels $(10-100 \mathrm{nM})$ against a huge (20,000-fold) $\mathrm{Ca}^{2+}$ concentration gradient in intracellular pools (known as $\mathrm{Ca}^{2+}$ stores, which are endowed with a $\left.\left[\mathrm{Ca}^{2+}\right]>100 \mu \mathrm{M}\right)$ [58]. The regulation of cytosolic and organelle $\mathrm{Ca}^{2+}$ levels is achieved by the orchestrated action of an extensive signaling machinery that comprises proteins able to either pump $\mathrm{Ca}^{2+}$ out from the cytosol to the extracellular space or into intracellular stores [59]. The most important intracellular organelles to be associated with $\mathrm{Ca}^{2+}$ handling in presynaptic neurons are 
the ER and mitochondria.

Studies on cultured sympathetic neurons from the stellate ganglion and superior cervical ganglion from the SHR have demonstrated that the depolarization-induced rise in intracellular free $\mathrm{Ca}^{2+}$ is significantly larger, and the decay of the $\mathrm{Ca}^{2+}$ transient also faster in the diseased neurons compared with neurons from age-matched normotensive Wistar Kyoto (WKY) rats[25, 60] (see Fig. 3A). N-type voltage-gated $\mathrm{Ca}^{2+}\left(\mathrm{Ca}_{\mathrm{v} 2.2}\right)$ channel activity is also enhanced in stellate neurons from a rat model of heart failure[30], and inhibition of this channel restores cardiac autonomic balance and prevents lethal arrhythmias[61]. In addition, we[62] also observed an enhanced wholecell $\mathrm{Ca}^{2+}$ current from the prehypertensive SHR postganglionic stellate neurons that was predominately blocked by $\omega$-conotoxin GVIA (Figure 3. B-D), supporting a role for the overactivity of $\mathrm{N}$-type $\mathrm{Ca}^{2+}$ channels contributing to abnormally high $\mathrm{Ca}^{2+}$ transients. Moreover, this suggests that channel dysregulation is an early molecular event that is highly conserved over several developmental stages from the prediseased phenotype to advanced heart failure[21].

\subsection{Dysregulation of endoplasmic reticulum in presynaptic neurons}

Various different factors may induce ER dysfunction, including age (decrease of ER calcium pump activity with age), metabolic stress, and genetic defects. Cell injury may develop under conditions where ER calcium homeostasis and/or folding or processing of proteins is disturbed [63]. The structure of the ER is highly conserved in neurons. The ER harbors the largest surface area of all eukaryotic organelles, and may represent up to 10 times the surface area of the plasma membrane[64]. Indeed, transmission electron microscopy images from stellate ganglia of 4-weekold SHR and WKY rats shows a striking difference in ER structure and organization[65]. In SHR rats, the ER is organized into spatially compact sheets compared with the more disperse and varied ER form observed in WKY neurons, suggesting that structural changes in neurons might underpin ER $\mathrm{Ca}^{2+}$ handling differences observed cellular phenotypes. Impaired ER proteostasis, involving $\mathrm{Ca}^{2+}$ signaling, protein synthesis, processing, trafficking, and degradation, is now recognized as a key risk factor in the pathogenesis of neurological disorders[66]. Disturbance of $\mathrm{ER} \mathrm{Ca}^{2+}$ homeostasis is commonly observed in severe neurodegenerative diseases $[67,68]$ and sympathetic hyperactivity diseases such as heart failure[69] and hypertension[70]. Reduced sarcoplasmic reticulum (SR) function is present in various forms of hypertrophy and heart failure where the SR plays an important role in cardiac contractility [71]. Pathological diastolic SR $\mathrm{Ca}^{2+}$ leak via calstabin2-depleted leaky RyR2 has been recognized as an important contributor to altered $\mathrm{Ca}^{2+}$ handling in heart failure[72], and the downregulation of SERCA2a is regarded as permissive for pulmonary vascular remodeling and the development of pulmonary arterial hypertension[73].

We have recently discovered that upregulating SERCA2a in normal rat stellate neurons transfected with Ad- mCherry-hATP2Aa (SERCA2a) leads to greater depolarization-induced $\mathrm{Ca}^{2+}$ transients. The time taken for the peak of the $\left[\mathrm{Ca}^{2+}\right]_{i}$ to fall by $50 \%$ was also significantly shorter (Fig. 4A) when compared with neurons transfected with the empty vector. Furthermore, stellate neurons from SHRs have a greater ER calcium load and greater abundance of ER per unit cell volume compared to normal neurons[65]. These results are consistent with previous observations in superior cervical ganglia neurons from young prehypertensive SHR[25]. This may be related to the activity of the SERCA transporter that is under the control of phospholamban (PLN), a small phosphoprotein that regulates the activity of SERCA. Expression of phosphorylated PLN (Ser16) is reduced in prehypertensive SHR superior cervical ganglion neurons when compared with total PLN. Therefore, less dephosphorylated PLN may increase SERCA activity, resulting in more rapid 
reuptake of calcium into the ER and faster recovery of the intracellular calcium transient in the prehypertensive SHR[25].

\subsection{Dysregulation of mitochondria in presynaptic neurons}

Much attention over the last decade has focused on the involvement of mitochondria in $\mathrm{Ca}^{2+}$ homeostasis where several comprehensive reviews on this topic have appeared [3, 74-76]. Since the late 1960s, it was established that mitochondria have the capacity to accumulate $\mathrm{Ca}^{2+}$ in an energy-dependent way[77]. Mitochondria participate both in $\mathrm{Ca}^{2+}$ buffering and in the formation of special communications with the ER. $\mathrm{Ca}^{2+}$ regulates the ability of mitochondria to provide ATP to energy-demanding processes, including pumps that mediate its removal from the cytosol[4]. They are also involved in buffering cytosolic calcium rises[25], uptake of ER-released $\mathrm{Ca}^{2+}$, and regulating neuronal excitability[78]. Functional or direct coupling of the ER and mitochondria in many cell types, including sympathetic neurons is now gaining support [68, 69].

In heart failure, increased mitochondrial $\mathrm{Ca}^{2+}$ concentrations alter the mitochondrial membrane potential, with elevated mitochondrial $\mathrm{Ca}^{2+}$ levels being linked to impaired mitochondrial energetics[79] and increased oxidative stress within cardiomyocytes[80, 81]. Chronic stimulation of $\beta$-adrenergic receptors has been directly coupled to mitochondrial reactive oxygen species (ROS) production through adrenergic receptor-mediated second messenger signalling[82, 83]. ROSmediated initiation of mitochondria-dependent cell death cascades has been repeatedly observed after chronic sympathetic activation, leading to overall declines in mitochondrial function[84, 85]. Lower mitochondrial membrane potential has been observed in young SHR sympathetic neurons[25]. This resulted in impaired mitochondrial $\mathrm{Ca}^{2+}$ uptake and release, which might partly be responsible for the increased $\left[\mathrm{Ca}^{2+}\right]_{\mathrm{i}}$ transient and faster decay in SHR sympathetic neurons (Figure 4B). Taken together, impaired mitochondrial function seems to be an important therapeutic target to restore autonomic balance, especially in heart failure and hypertension. In particular, the role of localized phosphodiesterases linked to mitochondrial cAMP signalling (Fig. 1) is thought to protect against apoptosis[86].

\section{Phosphodiesterase signaling and expression in cardiac dysautonomia}

Phosphodiesterases (PDEs) are currently receiving much attention as possible pharmacotherapeutic targets for treatment of cardiovascular disease, in particular targeting cardiac autonomic responses[21]. There are 11 subfamilies of PDE comprising about 21 different genes each containing several splice variants and isoforms making up more than a hundred specific human PDEs[87]. PDE catalyze the hydrolysis of cAMP and cGMP by controlling their rates of degradation, thereby regulating the intracellular concentrations of the cyclic nucleotide [88].

PDE1, PDE2, PDE3, PDE10, and PDE11 possess dual specificity, acting on both cAMP and cGMP. PDE2 and 3 are expressed in both sympathetic neurons and cardiomyocytes[62, 89]. PDE3 is encoded by two genes, PDE3A and PDE3B. PDE3A is responsible for the inotropic and chronotropic effects of PDE3 inhibitors, as it associates with PLB in a phosphorylation-dependent manner, controls SERCA2 activity and $\mathrm{Ca}^{2+}$ reuptake in the SR[90, 91]. Moreover, PDE3A is associated with myocyte apoptosis, likely through sustained induction of inducible cAMP early repressor (ICER)[92], leading to the hypothesis that its overexpression may limit cardiac damages. It was recently described that in the treatment of patients with heart failure, PDE3 inhibitors were effective in the acute setting, however, they were associated with increased sudden cardiac death 
following long-term administration, possibly reflecting pro-apoptotic and pro-hypertrophic consequences of increased cAMP-mediated signaling in cardiac myocytes[93]. Our work recently demonstrated a switching from cGMP acting via PDE3 in the control sympathetic neurons to PDE2A in the prohypertensive SHR neurons in the modulation of the $\mathrm{N}$-type $\mathrm{Ca}^{2+}$ current. Suggesting that a disturbance in the regulation of PDE-coupled cyclic nucleotides linked to N-type $\mathrm{Ca}^{2+}$ channels is an early hallmark of the prohypertensive phenotype associated with intracellular $\mathrm{Ca}^{2+}$ impairment underpinning sympathetic dysautonomia [62].

PDE2A has been shown to play important roles in many signal transduction pathways as a regulator of both cGMP and cAMP levels. There is growing interest in PDE2A inhibitors as potential agents for cognitive enhancement[94], and evidence suggests that these agents may enhance synaptic plasticity via presynaptic modulation of cAMP hydrolysis[95]. Chronic stress affects NMDAR-dependent nNOS and CaMKII production at hippocampal synapses, thereby resulting in enhanced neuronal PDE2A activity. Enhanced PDE2A activity accelerates the hydrolysis of cAMP/cGMP, resulting in overall impairment of learning and memory processes. Emerging evidence suggests inhibition of PDE2A can reversed impaired cognition and neuronal remodeling[96].

Three PDE2A splice variants have been identified, PDE2A1, PDE2A2 \& PDE2A3, and they are expressed in a wide variety of cells and tissues, including the heart, platelets, sympathetic nerve, and endothelium[87, 97]; PDE2A1 is cytosolic whereas PDE2A2 (rat only) and PDE2A3 are membrane bound[97]. Monterisi and co-workers[86] demonstrated that in rat cardiac myocytes and other cell types, mitochondrial PDE2A2 regulates local cAMP levels and PKA-dependent phosphorylation. Instead of regulating mitochondrial metabolism, this pool of cAMP influences mitochondrial dynamics and protects them from ionomycin-induced apoptosis. Another interesting piece of research recently reported that the expression of PDE2A was up-regulated in hearts from mice undergoing abdominal aortic constriction and blockade of PDE2A promotes cGMP, but not cAMP signaling to offset the pathogenesis of experimental heart failure, reversing the development of left ventricular hypertrophy, compromised contractility, and cardiac fibrosis (Figure 5A) [98]. This beneficial effect of PDE2A inhibition was dependent on endogenous NO bioactivity and stimulation of GC-1 in murine hearts, but not natriuretic peptides.

Indeed PDE2A overexpression in normal stellate neurons blunts the activity of brain natriuretic peptide (BNP) by decreasing cGMP production. This negatively affects downstream effectors such as the neuronal $\mathrm{Ca}^{2+}$ current, intracellular $\mathrm{Ca}^{2+}$ transient resulting in greater neurotransmitter release[99]. Interestingly, PDE2A activity in the stellate ganglia is increased in both SHRs and patients with sympathetic hyperactivity compared with their respective controls[26], suggesting that these levels may negate the beneficial action of BNP by hydrolyzing cGMP. Specifically, we found BNP increased cGMP levels in a dose-dependent manner as expected in healthy neurons, however, the response was markedly attenuated in diseased neurons (Figure 5B). The effect of BNP on the neuronal calcium current was also significantly reduced after 10-minute exposure in normal neurons, but BNP failed to affect the diseased neurons. The inhibitory action of BNP was restored following inhibition of PDE2A with Bay 60-7550, or following gene transfer of the dominant-negative of PDE2A in disease neurons. These results indicate that BNP loses its efficacy on postganglionic stellate sympathetic neurons due to over-activity of PDE2A, thereby impairing cGMP-PKG regulation of the neuronal calcium current and intracellular calcium transient, resulting in facilitated release of cardiac NE (Figure 6). 


\section{Conclusions and future directions}

Abnormality in cardiac sympathetic signaling is a key component in the etiology and progression of several cardiovascular diseases, yet the ability of the neuron to drive the myocyte phenotype in disease is not been firmly established. In this review, we have summarized the role of cyclic nucleotides in $\mathrm{Ca}^{2+}$ homeostasis and neurotransmission in sympathetic neurons, and discussed how they affect myocyte excitability in cardiovascular disease. In particular, we focused on the cyclic nucleotide-coupled PDE2A as a therapeutic target to rescue the BNP responsiveness in SHR stellate neurons. Recently, it has been reported that PDE2A2 is specifically targeted to the mitochondrial matrix, where it forms a local signaling system with soluble adenylyl cyclase regulating the respiratory chain[100] and mitochondria morphology[86]. Whether mitochondrial PDE2A2 is responsible for PDE2A overactivity that occurs in sympathetic neurons is states of dysautonomia remains to be established.

\section{Acknowledgements}

This work was supported by a BHF Programme Grant RG/17/14/33085.

\section{Figure Legends}

Fig. 1. Diagram of intracellular $\mathrm{Ca}^{2+}$ homeostasis and signal transduction pathways in cardiac sympathetic neurons linked to neurotransmission. An action potential leads to the opening of voltage-gated $\mathrm{Ca}^{2+}$ channels and transiently increases the local $\mathrm{Ca}^{2+}$ concentration. $\mathrm{Ca}^{2+}$ then triggers synaptic vesicle exocytosis. The potential signal transduction is modulated by natriuretic peptides (NP) and nitric oxide (NO)-CAPON pathways that target cGMP, and adrenergic receptor (AR)/adenylyl cyclase (AC) that target cAMP, via PKG and PKA to cause inhibition and activation of the channel current. Phosphodiesterases (PDEs) catalyze the hydrolysis of the cyclic nucleotides (CN) cAMP and/or cGMP that in turn affects $\mathrm{Ca}^{2+}$ release from the endoplasmic reticulum (ER) and its buffering from the mitochondria.

Fig. 2. Co-cultures of sympathetic neurons and ventricular myocytes from the pro-hypertensive spontaneously hypertensive rata SHR animals are hyper-responsive when compared to the normotensive WKY. A. Cardiac sympathetic stellate neurons were plated on top of ventricular myocytes. Pro-hypertensive SHRnSHRm cultures were compared to normotensive WKYnWKYm cultures to test whether the sympathetic hyperactivity observed in the hypertensive state, translates into a co-culture model. To investigate which cell is the primary driver behind this cardiac autonomic phenotype, two cross-cultures (SHRn/WKYm \& WKYn/SHRm) were developed and exposed to the same experimental protocols. B. Immunofluoresence staining confirmed the cellular phenotype. Sarcomeric alpha actinin marks the myocytes (red), Tyrosine hydroxylase (TH -green) was present in sympathetic neurons. Note the neuronal processes interweave with the myocytes resulting in a rich innervation of the myocytes. C-F. The myocytes of the pre-hypertensive SHR and WKY co-cultures were selectively infected with the Ad-Epac-SH187 cAMP FRET sensor. 
The neurons in the co-culture were stimulated using $10 \mu \mathrm{M}$ nicotine to stimulate sympathetic neurotransmission. The myocytes from the SHRnSHRm co-cultures (D) had significantly higher cAMP responses to $10 \mu \mathrm{M}$ nicotine stimulation when compared to the WKYnWKYm cultures (C). The nicotine evoked cAMP responses in the WKYnSHRm were significantly smaller compared to the SHRnSHRm, and not significantly different from that of the WKYnWKYm cultures (E). The cAMP levels of the SHRnWKYm were substantially (though not significantly) larger than those of the WKYnWKYm. This response was not significantly different from the diseased SHRnSHRm culture suggesting a partial recapitulation of the diseased phenotype (F). Modified from Larsen et al. (2016) with permission.

Fig. 3. Enhanced intracellular $\mathrm{Ca}^{2+}$ transient and current from the pro-hypertensive SHR sympathetic neurons. A: Raw data trace recording intracellular calcium transient $\left(\left[\mathrm{Ca}^{2+}\right]_{\mathrm{i}}\right)$ in a single cardiac sympathetic neuron from 4-week-old WKY rats and SHRs. A neuron loaded with fura-2 acetoxymethyl ester (Fura-2/AM, $2 \mu \mathrm{mol} / \mathrm{l}$ ) was stimulated by $50 \mathrm{mmol} / \mathrm{l} \mathrm{KCl}$ for 30 seconds to depolarize the neuron and evoke voltage-gated $\mathrm{Ca}^{2+}$ entry. $\mathrm{Ca}^{2+}$ concentration were colour-coded with a low $\mathrm{Ca}^{2+}$ concentration in blue and a high $\mathrm{Ca}^{2+}$ concentration in red. $\mathbf{B}$ : Representative peak $(-10 \mathrm{mV})$ voltage-clamp traces of WKY and SHR in normal conditions (solid lines) and after treatment with conotoxin GVIA (dashed lines). C: Current-voltage relationship showing uniform reduction in both the SHR and WKY neurons at multiple voltages after treatment with conotoxin GVIA. The peak current remains at $-10 \mathrm{mV}$ and was not significantly different between the WKY and SHR. D: Bar chart of the peak currents $(-10 \mathrm{mV})$ showing a $75 \%$ (control) and $83 \%$ (SHR) reduction of the $\mathrm{N}$-type $\mathrm{Ca}^{2+}$ current down to levels that were not significantly different between the strains after treatment with conotoxin GVIA. Dashed lines represent the mean of the WKY (black) and SHR (red) control data. Data are represented as the mean \pm SEM. Modified from Lu et al. (2015) and Larsen et al. (2016a) with permission.

Fig. 4. Contribution of endoplasmic reticulum (A) and mitochondria (B) to intracellular $\mathrm{Ca}^{2+}$ transient in sympathetic neurons. Aa: Ad-mCherry-SERCA2a transfected stellate ganglia neurons from (4 to 5 wk, 90-120 g) Sprague-Dawley (SD) rat. (i) Bright field image, (ii) composite, and (iii) excitation at $587 \mathrm{~nm}$ to excite mCherry fluorescent tag. Only cells expressing mCherry fluorescence were used for experiments. Ab: Example raw data trace from isolated stellate ganglia neurons of the young SD rat (gray line, Ad-mCherry [empty]; black line, Ad-mCherry-SERCA2a) exposed to $50 \mathrm{mmol} / \mathrm{L}$ of $\mathrm{KCl}(30 \mathrm{~s})$ to depolarize the neuron resulting in an increase in $\left[\mathrm{Ca}^{2+}\right]_{\mathrm{i}}$. Ac: Group mean data showing peak depolarization evoked intracellular free $\mathrm{Ca}^{2+}$ increase between empty $(\mathrm{n}=57)$ and SERCA2a $(\mathrm{n}=68)$ transfected stellate neurons. Ad: Group mean data of 50\% fall time of $\left(\left[\mathrm{Ca}^{2+}\right]_{\mathrm{i}}\right)$ from the peak (empty, $\mathrm{n}=37$; SERCA2a, $\left.\mathrm{n}=42\right) .{ }^{*} \mathrm{P}<0.05$. Ba: Typical experimental protocol showing fura-2/AM fluorescence ratio to assess the contribution of mitochondria to $\left[\mathrm{Ca}^{2+}\right]_{\mathrm{i}}$ in superior cervical ganglion (SCG) neurons from young SHRs and WKY rats. Bb: Statistical data showing the peak evoked $\left[\mathrm{Ca}^{2+}\right]_{\mathrm{i}}$ both before (S1) and after (S2) mitochondrial $\mathrm{Ca}^{2+}$ store depletion with FCCP between young SHRs and WKY rats. Bc, Time taken for the $\left[\mathrm{Ca}^{2+}\right]_{\mathrm{i}}$ from peak to $50 \%$ and $10 \%$ of the increase (triangles, $50 \%$ decay time; circles, $90 \%$ decay time) after high $\mathrm{K}^{+}$challenge. $* \mathrm{P}<0.05,{ }^{* *} \mathrm{P}<0.01$, ${ }^{* * *} \mathrm{P}<0.001$. Modified from Shanks et al. (2017) and Li et al. (2012) with permission.

Fig. 5. A: PDE2A overexpression in hypertrophied hearts affects cGMP but not cAMP levels. Immunoblot analysis of PDE2A expression (a), cardiac cGMP (b) and cAMP (c) levels in wholeheart homogenates from mice subjected to abdominal aortic constriction (AAC) for $6 \mathrm{w}$ in the 
absence and presence of PDE2 inhibition (PDEi) with BAY 60-7550 (10 mg. $\mathrm{kg}^{-1} \cdot \mathrm{d}^{-1} \mathrm{p} .0$. , initiated at 3 wk). Data are expressed as mean \pm SEM with analysis by one-way ANOVA with a Bonferroni post hoc test. $* \mathrm{P}<0.05,{ }^{*} \mathrm{P}<0.01$ versus sham; \#P $<0.05$ versus AAC. Modified from Baliga et al. (2018) with permission. B: BNP receptor activated cGMP levels attenuated in SHR neurons were associated with enhanced PDE2A activity (Lui et al. 2018). Representative data traces showing dynamics of cytosolic cGMP-induced fluorescence resonance energy transfer (FRET) changes by ratiometric recording of CFP and YFP emission changes in response to increasing concentrations of brain natriuretic peptide (BNP) in spontaneously hypertensive rat (SHR) and Wistar cardiac sympathetic neurons. Saturation of the sensor was achieved using 3morpholinosydnonimine chloride (SIN-1 chloride; $10 \mu \mathrm{mol} / \mathrm{l})+$ IBMX (100 $\mu \mathrm{mol} / \mathrm{l})$. C: Simplified schematic of BNP induced effects in cardiac sympathetic neurons. BNP binding to the natriuretic peptide receptor-A (NPR-A) stimulate cyclic GMP (cGMP) production by activation of the particulate guanylyl cyclase (pGC). cGMP modulates the activity of cGMP-dependent protein kinase $\mathrm{G}$ (PKG) to exert its biological actions, also regulates PDE2A and cation channels. The cGMP signal was terminated by a PDE2A that hydrolyze cGMP to 5'-GMP. Modified from Liu et al. (2018) with permission.

Fig. 6. Schematic representation depicting the effect of PDE2A on the regulation of BNP in cardiac neurotransmission in normal and diseased neurons. A: Brain natriuretic peptide (BNP) can decrease neurotransmission in healthy neurons by activating the particulate guanylyl cyclasecoupled (pGC coupled) cGMP pathway to decrease intracellular calcium transients and exocytosis resulting in a decrease in the heart rate (HR) response to sympathetic nerve stimulation. Overexpression of PDE2A in healthy neurons mimics the lack of BNP efficacy seen in diseased neurons. B: In the diseased neuron, PDE2A is increased and increases the hydrolysis of cGMP and decreases the sympatholytic action of BNP that results in an increase in neurotransmission and HR responses to sympathetic activation. Blockade of overexpressed PDE2A with Bay 60-7550 or overexpression of catalytically inactive PDE2A reestablished the modulatory action of BNP in the diseased neuron. $\beta 1$-AR, $\beta 1$-adrenergic receptor; NE, norepinephrine; NPR, natriuretic peptide receptor; SNS, sympathetic nerve stimulation. Modified from Liu et al. (2018) with permission.

\section{References}

[1] S. Ringer, A further Contribution regarding the influence of the different Constituents of the Blood on the Contraction of the Heart, J Physiol 4(1) (1883) 29-42.3.

[2] M.J. Berridge, Calcium signalling remodelling and disease, Biochem Soc Trans 40(2) (2012) 297-309.

[3] M. Brini, T. Cali, D. Ottolini, E. Carafoli, Neuronal calcium signaling: function and dysfunction, Cell Mol Life Sci 71(15) (2014) 2787-814.

[4] R. Bagur, G. Hajnoczky, Intracellular $\mathrm{Ca}(2+)$ Sensing: Its Role in Calcium Homeostasis and Signaling, Mol Cell 66(6) (2017) 780-788.

[5] W.A. Catterall, Structure and regulation of voltage-gated Ca2+ channels, Annu Rev Cell Dev Biol 16 (2000) 521-55.

[6] G.W. Zamponi, J. Striessnig, A. Koschak, A.C. Dolphin, The Physiology, Pathology, and Pharmacology of Voltage-Gated Calcium Channels and Their Future Therapeutic Potential, Pharmacol Rev 67(4) (2015) 821-70. 
[7] N. Tohse, N. Sperelakis, cGMP inhibits the activity of single calcium channels in embryonic chick heart cells, Circ Res 69(2) (1991) 325-31.

[8] L.H. Jiang, D.J. Gawler, N. Hodson, C.J. Milligan, H.A. Pearson, V. Porter, D. Wray, Regulation of cloned cardiac L-type calcium channels by cGMP-dependent protein kinase, J Biol Chem 275(9) (2000) 6135-43.

[9] M. Conti, J. Beavo, Biochemistry and physiology of cyclic nucleotide phosphodiesterases: essential components in cyclic nucleotide signaling, Annu Rev Biochem 76 (2007) 481-511.

[10] M. Zaccolo, M.A. Movsesian, CAMP and cGMP signaling cross-talk: role of phosphodiesterases and implications for cardiac pathophysiology, Circ Res 100(11) (2007) 1569-78.

[11] A. Stangherlin, M. Zaccolo, cGMP-cAMP interplay in cardiac myocytes: a local affair with farreaching consequences for heart function, Biochem Soc Trans 40(1) (2012) 11-4.

[12] A. Stangherlin, F. Gesellchen, A. Zoccarato, A. Terrin, L.A. Fields, M. Berrera, N.C. Surdo, M.A. Craig, G. Smith, G. Hamilton, M. Zaccolo, cGMP signals modulate cAMP levels in a compartment-specific manner to regulate catecholamine-dependent signaling in cardiac myocytes, Circ Res 108(8) (2011) 92939.

[13] S.H. Francis, M.A. Blount, J.D. Corbin, Mammalian cyclic nucleotide phosphodiesterases: molecular mechanisms and physiological functions, Physiol Rev 91(2) (2011) 651-90.

[14] S.H. Francis, J.L. Busch, J.D. Corbin, D. Sibley, cGMP-dependent protein kinases and cGMP phosphodiesterases in nitric oxide and cGMP action, Pharmacol Rev 62(3) (2010) 525-63.

[15] M.J. Shen, D.P. Zipes, Role of the autonomic nervous system in modulating cardiac arrhythmias, Circ Res 114(6) (2014) 1004-21.

[16] N. Herring, Paterson, D.J., Levick's Introduction to Cardiovascular Physiology CRC Press; 6 edition, Boca Raton, Florida, United States, 2018.

[17] R.D. Harvey, A.E. Belevych, Muscarinic regulation of cardiac ion channels, Br J Pharmacol 139(6) (2003) 1074-84.

[18] L. Wang, M. Henrich, K.J. Buckler, M. McMenamin, C.J. Mee, D.B. Sattelle, D.J. Paterson, Neuronal nitric oxide synthase gene transfer decreases [Ca2+]i in cardiac sympathetic neurons, J Mol Cell Cardiol 43(6) (2007) 717-25.

[19] E.J. Danson, D. Li, L. Wang, T.A. Dawson, D.J. Paterson, Targeting cardiac sympatho-vagal imbalance using gene transfer of nitric oxide synthase, J Mol Cell Cardiol 46(4) (2009) 482-9.

[20] N. Herring, D.J. Paterson, Neuromodulators of peripheral cardiac sympatho-vagal balance, Exp Physiol 94(1) (2009) 46-53.

[21] D. Li, D.J. Paterson, Cyclic nucleotide regulation of cardiac sympatho-vagal responsiveness, J Physiol (2016) DOI: 10.1113/JP271827.

[22] A. Ali, H. Holm, J. Molvin, E. Bachus, G. Tasevska-Dinevska, A. Fedorowski, A. Jujic, M. Magnusson, Autonomic dysfunction is associated with cardiac remodelling in heart failure patients, ESC Heart Fail 5(1) (2018) 46-52.

[23] D.Y. Zhang, A.S. Anderson, The sympathetic nervous system and heart failure, Cardiol Clin 32(1) (2014) 33-45, vii.

[24] S. Abdul-Ghani, A.N. Fleishman, I. Khaliulin, M. Meloni, G.D. Angelini, M.S. Suleiman, Remote ischemic preconditioning triggers changes in autonomic nervous system activity: implications for cardioprotection, Physiol Rep 5(3) (2017).

[25] D. Li, C.W. Lee, K. Buckler, A. Parekh, N. Herring, D.J. Paterson, Abnormal intracellular calcium homeostasis in sympathetic neurons from young prehypertensive rats, Hypertension 59(3) (2012) 642-9. [26] K. Liu, D. Li, G. Hao, D. McCaffary, O. Neely, L. Woodward, D. Ioannides, C.J. Lu, M. Brescia, M. Zaccolo, H. Tandri, O.A. Ajijola, J.L. Ardell, K. Shivkumar, D.J. Paterson, Phosphodiesterase 2A as a therapeutic target to restore cardiac neurotransmission during sympathetic hyperactivity, JCl Insight 3(9) (2018). 
[27] J.A. Armour, D.A. Murphy, B.X. Yuan, S. Macdonald, D.A. Hopkins, Gross and microscopic anatomy of the human intrinsic cardiac nervous system, Anat Rec 247(2) (1997) 289-98.

[28] D.H. Pauza, V. Skripka, N. Pauziene, R. Stropus, Morphology, distribution, and variability of the epicardiac neural ganglionated subplexuses in the human heart, Anat Rec 259(4) (2000) 353-82.

[29] B.J. Pardini, D.D. Lund, P.G. Schmid, Organization of the sympathetic postganglionic innervation of the rat heart, J Auton Nerv Syst 28(3) (1989) 193-201.

[30] H. Tu, J. Liu, D. Zhang, H. Zheng, K.P. Patel, K.G. Cornish, W.Z. Wang, R.L. Muelleman, Y.L. Li, Heart failure-induced changes of voltage-gated $\mathrm{Ca} 2+$ channels and cell excitability in rat cardiac postganglionic neurons, Am J Physiol Cell Physiol 306(2) (2014) C132-42.

[31] P.J. Schwartz, M.T. La Rovere, G.M. De Ferrari, D.L. Mann, Autonomic modulation for the management of patients with chronic heart failure, Circ Heart Fail 8(3) (2015) 619-28.

[32] O.A. Ajijola, D. Yagishita, N.K. Reddy, K. Yamakawa, M. Vaseghi, A.M. Downs, D.B. Hoover, J.L. Ardell, K. Shivkumar, Remodeling of stellate ganglion neurons after spatially targeted myocardial infarction: Neuropeptide and morphologic changes, Heart Rhythm 12(5) (2015) 1027-35.

[33] G. Grassi, A. Mark, M. Esler, The sympathetic nervous system alterations in human hypertension, Circ Res 116(6) (2015) 976-90.

[34] B.A. Habecker, M.E. Anderson, S.J. Birren, K. Fukuda, N. Herring, D.B. Hoover, H. Kanazawa, D.J. Paterson, C.M. Ripplinger, Molecular and cellular neurocardiology: development, and cellular and molecular adaptations to heart disease, J Physiol 594(14) (2016) 3853-75.

[35] H.L. Haas, O.A. Sergeeva, O. Selbach, Histamine in the nervous system, Physiol Rev 88(3) (2008) 1183-241.

[36] D. Li, N. Nikiforova, C.J. Lu, K. Wannop, M. McMenamin, C.W. Lee, K.J. Buckler, D.J. Paterson, Targeted neuronal nitric oxide synthase transgene delivery into stellate neurons reverses impaired intracellular calcium transients in prehypertensive rats, Hypertension 61(1) (2013) 202-7.

[37] M. Klapholz, Beta-blocker use for the stages of heart failure, Mayo Clin Proc 84(8) (2009) 718-29.

[38] P.J. Schwartz, Cardiac sympathetic denervation to prevent life-threatening arrhythmias, Nat Rev Cardiol 11(6) (2014) 346-53.

[39] W.F. Lubbe, T. Podzuweit, L.H. Opie, Potential arrhythmogenic role of cyclic adenosine monophosphate (AMP) and cytosolic calcium overload: implications for prophylactic effects of betablockers in myocardial infarction and proarrhythmic effects of phosphodiesterase inhibitors, J Am Coll Cardiol 19(7) (1992) 1622-33.

[40] L.H. Opie, W.T. Clusin, Cellular mechanism for ischemic ventricular arrhythmias, Annu Rev Med 41 (1990) 231-8.

[41] R.E. Kleiger, J.P. Miller, J.T. Bigger, Jr., A.J. Moss, Decreased heart rate variability and its association with increased mortality after acute myocardial infarction, Am J Cardiol 59(4) (1987) 256-62.

[42] M.T. La Rovere, J.T. Bigger, Jr., F.I. Marcus, A. Mortara, P.J. Schwartz, Baroreflex sensitivity and heart-rate variability in prediction of total cardiac mortality after myocardial infarction. ATRAMI (Autonomic Tone and Reflexes After Myocardial Infarction) Investigators, Lancet 351(9101) (1998) 47884.

[43] J. Nolan, P.D. Batin, R. Andrews, S.J. Lindsay, P. Brooksby, M. Mullen, W. Baig, A.D. Flapan, A. Cowley, R.J. Prescott, J.M. Neilson, K.A. Fox, Prospective study of heart rate variability and mortality in chronic heart failure: results of the United Kingdom heart failure evaluation and assessment of risk trial (UK-heart), Circulation 98(15) (1998) 1510-6.

[44] R.F. Leighton, T.D. Fraker, Jr., Silent myocardial ischemia: concern is justified for the patient with known coronary artery disease, Ann Intern Med 100(4) (1984) 599-601.

[45] D. Ely, A. Caplea, G. Dunphy, H. Daneshvar, M. Turner, A. Milsted, M. Takiyyudin, Spontaneously hypertensive rat $\mathrm{Y}$ chromosome increases indexes of sympathetic nervous system activity, Hypertension 29(2) (1997) 613-8. 
[46] H. Yemane, M. Busauskas, S.K. Burris, M.M. Knuepfer, Neurohumoral mechanisms in deoxycorticosterone acetate (DOCA)-salt hypertension in rats, Exp Physiol 95(1) (2010) 51-5.

[47] D.A. Heaton, M. Lei, D. Li, S. Golding, T.A. Dawson, R.M. Mohan, D.J. Paterson, Remodeling of the cardiac pacemaker L-type calcium current and its beta-adrenergic responsiveness in hypertension after neuronal NO synthase gene transfer, Hypertension 48(3) (2006) 443-52.

[48] M.S. Rumantir, D.M. Kaye, G.L. Jennings, M. Vaz, J.A. Hastings, M.D. Esler, Phenotypic evidence of faulty neuronal norepinephrine reuptake in essential hypertension, Hypertension 36(5) (2000) 824-9.

[49] C. Zugck, D. Lossnitzer, J. Backs, A. Kristen, R. Kinscherf, M. Haass, Increased cardiac norepinephrine release in spontaneously hypertensive rats: role of presynaptic alpha-2A adrenoceptors, J Hypertens 21(7) (2003) 1363-9.

[50] J. Shanks, S. Manou-Stathopoulou, C.J. Lu, D. Li, D.J. Paterson, N. Herring, Cardiac sympathetic dysfunction in the pre-hypertensive spontaneously hypertensive rat, Am J Physiol Heart Circ Physiol (2013).

[51] F. Yanowitz, J.B. Preston, J.A. Abildskov, Functional distribution of right and left stellate innervation to the ventricles. Production of neurogenic electrocardiographic changes by unilateral alteration of sympathetic tone, Circ Res 18(4) (1966) 416-28.

[52] A.Y. Tan, S. Zhou, B.C. Jung, M. Ogawa, L.S. Chen, M.C. Fishbein, P.S. Chen, Ectopic atrial arrhythmias arising from canine thoracic veins during in vivo stellate ganglia stimulation, Am J Physiol Heart Circ Physiol 295(2) (2008) H691-8.

[53] G.A. Ng, R. Mantravadi, W.H. Walker, W.G. Ortin, B.R. Choi, W. de Groat, G. Salama, Sympathetic nerve stimulation produces spatial heterogeneities of action potential restitution, Heart Rhythm 6(5) (2009) 696-706.

[54] R. Arora, Recent insights into the role of the autonomic nervous system in the creation of substrate for atrial fibrillation: implications for therapies targeting the atrial autonomic nervous system, Circ Arrhythm Electrophysiol 5(4) (2012) 850-9.

[55] M. Franzoso, T. Zaglia, M. Mongillo, Putting together the clues of the everlasting neuro-cardiac liaison, Biochim Biophys Acta 1863(7 Pt B) (2016) 1904-15.

[56] H.E. Larsen, K. Lefkimmiatis, D.J. Paterson, Sympathetic neurons are a powerful driver of myocyte function in cardiovascular disease, Sci Rep 6 (2016b) 38898.

[57] B. Katz, R. Miledi, lonic requirements of synaptic transmitter release, Nature 215(5101) (1967) 651.

[58] D.E. Clapham, Calcium signaling, Cell 131(6) (2007) 1047-58.

[59] T. Pozzan, R. Rizzuto, P. Volpe, J. Meldolesi, Molecular and cellular physiology of intracellular calcium stores, Physiol Rev 74(3) (1994) 595-636.

[60] C.J. Lu, G. Hao, N. Nikiforova, H.E. Larsen, K. Liu, M.J. Crabtree, D. Li, N. Herring, D.J. Paterson, CAPON modulates neuronal calcium handling and cardiac sympathetic neurotransmission during dysautonomia in hypertension, Hypertension 65(6) (2015) 1288-97.

[61] Y. Yamada, H. Kinoshita, K. Kuwahara, Y. Nakagawa, Y. Kuwabara, T. Minami, C. Yamada, J. Shibata, K. Nakao, K. Cho, Y. Arai, S. Yasuno, T. Nishikimi, K. Ueshima, S. Kamakura, M. Nishida, S. Kiyonaka, Y. Mori, T. Kimura, K. Kangawa, K. Nakao, Inhibition of N-type Ca2+ channels ameliorates an imbalance in cardiac autonomic nerve activity and prevents lethal arrhythmias in mice with heart failure, Cardiovasc Res 104(1) (2014) 183-93.

[62] H.E. Larsen, E.N. Bardsley, K. Lefkimmiatis, D.J. Paterson, Dysregulation of Neuronal Ca2+ Channel Linked to Heightened Sympathetic Phenotype in Prohypertensive States, J Neurosci 36(33) (2016a) 8562-73.

[63] W. Paschen, A. Frandsen, Endoplasmic reticulum dysfunction--a common denominator for cell injury in acute and degenerative diseases of the brain?, J Neurochem 79(4) (2001) 719-25. 
[64] A. Luarte, V.H. Cornejo, F. Bertin, J. Gallardo, A. Couve, The axonal endoplasmic reticulum: One organelle-many functions in development, maintenance, and plasticity, Dev Neurobiol 78(3) (2018) 181208.

[65] J. Shanks, Herring H, Johnson E, Liu K, Li D, Paterson DJ, Overexpression of SERCA2a promotes cardiac sympathetic neurotransmission via abnormal endoplasmic reticulum and mitochondria $\mathrm{Ca} 2+$ regulation, Hypertension April (2017).

[66] J. Jung, M. Michalak, L.B. Agellon, Endoplasmic Reticulum Malfunction in the Nervous System, Front Neurosci 11 (2017) 220.

[67] W. Zeiger, K.S. Vetrivel, V. Buggia-Prevot, P.D. Nguyen, S.L. Wagner, M.L. Villereal, G. Thinakaran, $\mathrm{Ca} 2+$ influx through store-operated $\mathrm{Ca} 2+$ channels reduces Alzheimer disease beta-amyloid peptide secretion, J Biol Chem 288(37) (2013) 26955-66.

[68] I. Bezprozvanny, P.R. Hiesinger, The synaptic maintenance problem: membrane recycling, Ca2+ homeostasis and late onset degeneration, Mol Neurodegener 8 (2013) 23.

[69] A.R. Marks, Calcium cycling proteins and heart failure: mechanisms and therapeutics, J Clin Invest 123(1) (2013) 46-52.

[70] J. Aguero, K. Ishikawa, L. Hadri, C.G. Santos-Gallego, K.M. Fish, E. Kohlbrenner, N. Hammoudi, C. Kho, A. Lee, B. Ibanez, A. Garcia-Alvarez, K. Zsebo, B.A. Maron, M. Plataki, V. Fuster, J.A. Leopold, R.J. Hajjar, Intratracheal Gene Delivery of SERCA2a Ameliorates Chronic Post-Capillary Pulmonary Hypertension: A Large Animal Model, J Am Coll Cardiol 67(17) (2016) 2032-46.

[71] M. Meyer, S.U. Trost, W.F. Bluhm, H.J. Knot, E. Swanson, W.H. Dillmann, Impaired sarcoplasmic reticulum function leads to contractile dysfunction and cardiac hypertrophy, Am J Physiol Heart Circ Physiol 280(5) (2001) H2046-52.

[72] I.A. Hobai, B. O'Rourke, Decreased sarcoplasmic reticulum calcium content is responsible for defective excitation-contraction coupling in canine heart failure, Circulation 103(11) (2001) 1577-84.

[73] L. Hadri, R.G. Kratlian, L. Benard, B.A. Maron, P. Dorfmuller, D. Ladage, C. Guignabert, K. Ishikawa, J. Aguero, B. Ibanez, I.C. Turnbull, E. Kohlbrenner, L. Liang, K. Zsebo, M. Humbert, J.S. Hulot, Y. Kawase, R.J. Hajjar, J.A. Leopold, Therapeutic efficacy of AAV1.SERCA2a in monocrotaline-induced pulmonary arterial hypertension, Circulation 128(5) (2013) 512-23.

[74] M.J. Devine, J.T. Kittler, Mitochondria at the neuronal presynapse in health and disease, Nat Rev Neurosci 19(2) (2018) 63-80.

[75] S. Marchi, S. Patergnani, S. Missiroli, G. Morciano, A. Rimessi, M.R. Wieckowski, C. Giorgi, P. Pinton, Mitochondrial and endoplasmic reticulum calcium homeostasis and cell death, Cell Calcium 69 (2018) 62-72.

[76] R. Rizzuto, D. De Stefani, A. Raffaello, C. Mammucari, Mitochondria as sensors and regulators of calcium signalling, Nat Rev Mol Cell Biol 13(9) (2012) 566-78.

[77] C.S. Rossi, A.L. Lehninger, STOICHIOMETRIC RELATIONSHIPS BETWEEN ACCUMULATION OF IONS BY MITOCHONDRIA AND THE ENERGY-COUPLING SITES IN THE RESPIRATORY CHAIN, Biochem Z 338 (1963) 698-713.

[78] P. Vanden Berghe, J.L. Kenyon, T.K. Smith, Mitochondrial Ca2+ uptake regulates the excitability of myenteric neurons, J Neurosci 22(16) (2002) 6962-71.

[79] M.R. Duchen, A. Verkhratsky, S. Muallem, Mitochondria and calcium in health and disease, Cell Calcium 44(1) (2008) 1-5.

[80] J.M. Huss, D.P. Kelly, Mitochondrial energy metabolism in heart failure: a question of balance, J Clin Invest 115(3) (2005) 547-55.

[81] G. Santulli, W. Xie, S.R. Reiken, A.R. Marks, Mitochondrial calcium overload is a key determinant in heart failure, Proc Natl Acad Sci U S A 112(36) (2015) 11389-94. 
[82] B. Menon, P. Krishnamurthy, E. Kaverina, J.N. Johnson, R.S. Ross, M. Singh, K. Singh, Expression of the cytoplasmic domain of beta1 integrin induces apoptosis in adult rat ventricular myocytes (ARVM) via the involvement of caspase-8 and mitochondrial death pathway, Basic Res Cardiol 101(6) (2006) 485-93. [83] M.G. Rosca, C.L. Hoppel, Mitochondrial dysfunction in heart failure, Heart Fail Rev 18(5) (2013) 60722.

[84] M. Izem-Meziane, B. Djerdjouri, S. Rimbaud, F. Caffin, D. Fortin, A. Garnier, V. Veksler, F. Joubert, R. Ventura-Clapier, Catecholamine-induced cardiac mitochondrial dysfunction and mPTP opening: protective effect of curcumin, Am J Physiol Heart Circ Physiol 302(3) (2012) H665-74.

[85] D.A. Brown, J.B. Perry, M.E. Allen, H.N. Sabbah, B.L. Stauffer, S.R. Shaikh, J.G. Cleland, W.S. Colucci, J. Butler, A.A. Voors, S.D. Anker, B. Pitt, B. Pieske, G. Filippatos, S.J. Greene, M. Gheorghiade, Expert consensus document: Mitochondrial function as a therapeutic target in heart failure, Nat Rev Cardiol 14(4) (2017) 238-250.

[86] S. Monterisi, M.J. Lobo, C. Livie, J.C. Castle, M. Weinberger, G. Baillie, N.C. Surdo, N. Musheshe, A. Stangherlin, E. Gottlieb, R. Maizels, M. Bortolozzi, M. Micaroni, M. Zaccolo, PDE2A2 regulates mitochondria morphology and apoptotic cell death via local modulation of cAMP/PKA signalling, Elife 6 (2017).

[87] A.T. Bender, J.A. Beavo, Cyclic nucleotide phosphodiesterases: molecular regulation to clinical use, Pharmacol Rev 58(3) (2006) 488-520.

[88] D.H. Maurice, H. Ke, F. Ahmad, Y. Wang, J. Chung, V.C. Manganiello, Advances in targeting cyclic nucleotide phosphodiesterases, Nat Rev Drug Discov 13(4) (2014) 290-314.

[89] N. Pavlaki, V.O. Nikolaev, Imaging of PDE2- and PDE3-Mediated cGMP-to-cAMP Cross-Talk in Cardiomyocytes, J Cardiovasc Dev Dis 5(1) (2018).

[90] F. Ahmad, W. Shen, F. Vandeput, N. Szabo-Fresnais, J. Krall, E. Degerman, F. Goetz, E. Klussmann, M. Movsesian, V. Manganiello, Regulation of sarcoplasmic reticulum Ca2+ ATPase 2 (SERCA2) activity by phosphodiesterase 3A (PDE3A) in human myocardium: phosphorylation-dependent interaction of PDE3A1 with SERCA2, J Biol Chem 290(11) (2015) 6763-76.

[91] S. Beca, F. Ahmad, W. Shen, J. Liu, S. Makary, N. Polidovitch, J. Sun, S. Hockman, Y.W. Chung, M. Movsesian, E. Murphy, V. Manganiello, P.H. Backx, Phosphodiesterase type 3A regulates basal myocardial contractility through interacting with sarcoplasmic reticulum calcium ATPase type 2a signaling complexes in mouse heart, Circ Res 112(2) (2013) 289-97.

[92] B. Ding, J. Abe, H. Wei, H. Xu, W. Che, T. Aizawa, W. Liu, C.A. Molina, J. Sadoshima, B.C. Blaxall, B.C. Berk, C. Yan, A positive feedback loop of phosphodiesterase 3 (PDE3) and inducible cAMP early repressor (ICER) leads to cardiomyocyte apoptosis, Proc Natl Acad Sci U S A 102(41) (2005) 14771-6. [93] M. Movsesian, Novel approaches to targeting PDE3 in cardiovascular disease, Pharmacol Ther 163 (2016) 74-81.

[94] A.A. Trabanco, P. Buijnsters, F.J. Rombouts, Towards selective phosphodiesterase 2A (PDE2A) inhibitors: a patent review (2010 - present), Expert Opin Ther Pat 26(8) (2016) 933-46.

[95] D. Fernandez-Fernandez, H. Rosenbrock, K.S. Kroker, Inhibition of PDE2A, but not PDE9A, modulates presynaptic short-term plasticity measured by paired-pulse facilitation in the CA1 region of the hippocampus, Synapse 69(10) (2015) 484-96.

[96] Y. Xu, J. Pan, J. Sun, L. Ding, L. Ruan, M. Reed, X. Yu, J. Klabnik, D. Lin, J. Li, L. Chen, C. Zhang, H. Zhang, J.M. O'Donnell, Inhibition of phosphodiesterase 2 reverses impaired cognition and neuronal remodeling caused by chronic stress, Neurobiol Aging 36(2) (2015) 955-70.

[97] C. Russwurm, G. Zoidl, D. Koesling, M. Russwurm, Dual acylation of PDE2A splice variant 3: targeting to synaptic membranes, J Biol Chem 284(38) (2009) 25782-90.

[98] R.S. Baliga, M.E.J. Preedy, M.S. Dukinfield, S.M. Chu, A.A. Aubdool, K.J. Bubb, A.J. Moyes, M.A. Tones, A.J. Hobbs, Phosphodiesterase 2 inhibition preferentially promotes NO/guanylyl cyclase/cGMP 
signaling to reverse the development of heart failure, Proc Natl Acad Sci U S A 115(31) (2018) E7428e7437.

[99] D. Li, C.J. Lu, G. Hao, H. Wright, L. Woodward, K. Liu, E. Vergari, N.C. Surdo, N. Herring, M. Zaccolo, D.J. Paterson, Efficacy of B-Type Natriuretic Peptide Is Coupled to Phosphodiesterase 2A in Cardiac Sympathetic Neurons, Hypertension 66(1) (2015) 190-8.

[100] R. Acin-Perez, M. Russwurm, K. Gunnewig, M. Gertz, G. Zoidl, L. Ramos, J. Buck, L.R. Levin, J. Rassow, G. Manfredi, C. Steegborn, A phosphodiesterase 2A isoform localized to mitochondria regulates respiration, J Biol Chem 286(35) (2011) 30423-32. 